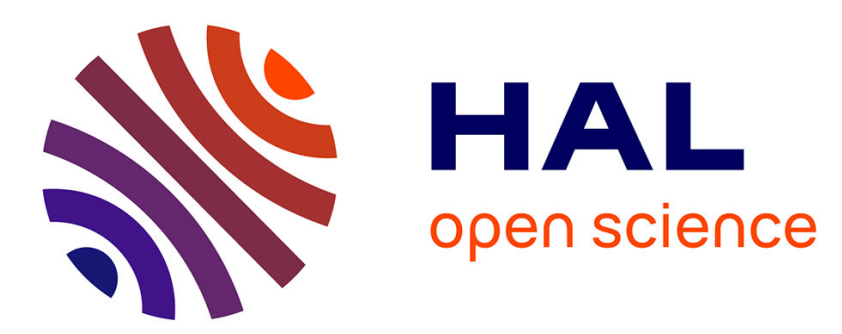

\title{
Influence of the interface on the optical activity of confined glucose films
}

Olivier Emile, Janine Emile, Aziz Ghoufi

\section{To cite this version:}

Olivier Emile, Janine Emile, Aziz Ghoufi. Influence of the interface on the optical activity of confined glucose films. Journal of Colloid and Interface Science, 2016, 477, pp.103-108. 10.1016/j.jcis.2016.05.047 . hal-01331955

\section{HAL Id: hal-01331955 \\ https://hal.science/hal-01331955}

Submitted on 17 Nov 2016

HAL is a multi-disciplinary open access archive for the deposit and dissemination of scientific research documents, whether they are published or not. The documents may come from teaching and research institutions in France or abroad, or from public or private research centers.
L'archive ouverte pluridisciplinaire HAL, est destinée au dépôt et à la diffusion de documents scientifiques de niveau recherche, publiés ou non, émanant des établissements d'enseignement et de recherche français ou étrangers, des laboratoires publics ou privés. 


\title{
Influence of the interface on the optical activity of confined glucose films
}

Olivier Emile ${ }^{\mathrm{a}, *}$, Janine Emile ${ }^{\mathrm{b}}$, Aziz Ghoufi ${ }^{\mathrm{b}}$

${ }^{a}$ Université de Rennes 1, Campus de Beaulieu, 35042 Rennes, France

bInstitut de Physique de Rennes, CNRS UMR 6521, Université de Rennes 1, Campus de Beaulieu, 35042 Rennes, France

\begin{abstract}
We report on the time evolution of the optical activity of a thinning liquid film containing glucose, and confined between two glass slides. This dynamics strongly depends on the presence of surfactant molecules. With sodium dodecylsulfate (SDS), we evidence favorable interactions of sugar molecules with the sulfate group. As previously observed for a freely suspended soap film in the air (see J. Colloid Interface Sci. 408 (2013) 113), this corresponds to an anchoring of glucose molecules at the interface. For glucose alone, we also highlight a molecular rearrangement that is not instantaneous and occurs after several minutes. This interfacial organization leads to an unusual giant optical activity that is different with or without SDS. Molecular simulations confirm the anchoring of the glucose molecules at the glass/liquid interface, and show a different molecular orientation in each case.
\end{abstract}

Keywords:

Sugar-containing film; optical activity; interfacial interaction; molecular simulations

*Corresponding author.

E-mail address: olivier.emile@univ-rennes1.fr (O. Emile) 


\section{Introduction}

Glucose and sugars in general play a central role in many scientific fields including chemistry [1-3], biology [4,5], medicine [6,7], pharmaceutics [8], as well as in the food industry [9-11]. Like most of the molecules of biological interest, glucose is a chiral molecule and thus its presence or its concentration could be detected via its optical activity either in solution or in its natural form [12-16]. This optical activity can sometimes be modified by the interaction with other molecules or with the environment [16-19]. In particular, we have recently reported a giant glucose optical activity in a draining sodium dodecylsulfate (SDS) film [20]. It has been interpreted as due to hydrogen bond interactions between the anionic group $-\mathrm{OSO}_{3}{ }^{-}$- of $\mathrm{SDS}$ molecules and the $\mathrm{OH}$ group of glucose molecules at the air/liquid interface. So, some questions may be issued: i) Does this giant effect saturate with thicker films? Indeed, since it is related to interfacial interactions, it should be insensitive to the bulk properties of the solution and plays a role for a very thin film only. ii) Are these properties due to the presence of SDS or could one find a similar effect on a film composed entirely of glucose? iii) If positive, is the glucose interface arrangement similar to the one with SDS? In order to address these two questions, one has to adapt the laser experimental set up of [20], since a liquid sugar-containing film is not stable. We have thus performed a series of experiments by confining a liquid film between two glass slides using glucose and also a mixture of glucose and SDS and studied its optical properties while thinning.

\section{Methods and materials}

\section{A. Experimental set-up}

To measure the thickness of the liquid film, we use an optical interference technique comparing the fringes from two different lasers. This method has been previously developed in [21]. It has been used either for free-standing soap films that guide light [22] or with functionalized particles [23]. Here, a red laser $(\lambda=633 \mathrm{~nm}$, Mlles Griot) and a green laser ( $\lambda=543 \mathrm{~nm}$, Mlles Griot) intercept a horizontal film confined between two glass slides (see figure 1). We deposit a liquid drop of volume $8 \mu \mathrm{l}$ on a plate, flattened by gravity and 
confined it with another slide so that the glass/liquid interfaces are sufficiently parallel for the quality of the transmitted light beams.

The film is either made of a solution (pure water) of glucose ( $\alpha$-D-Glucose, Sigma Aldrich, 96\% grade, concentration $100 \mathrm{~g} / \mathrm{L})$ in the case of sugar alone, or glucose (100 g/L) + SDS (Sigma Aldrich, 99.5\%, concentration $12 \mathrm{~g} / \mathrm{L}$ ) in the case of sugar + SDS. We measure the optical activity with a third laser $(\lambda=532 \mathrm{~nm}$, Crystal laser). We use here two polarizers $\left(\mathrm{P}_{1}\right.$ and $\left.\mathrm{P}_{2}\right)$ which orientations are not exactly orthogonal to each other. There is a small angle $\theta_{0}=20^{\circ}$ regarded to cross orientations. Then some light is passing through $\mathrm{P}_{2}$ in the absence of any active medium. With a chiral medium the rotation angle due to the optical activity should vary linearly with the intensity on the photodiode (D), see inset of figure 1 . Each experiment is repeated three times to check the reproducibility and in order to get a good statistics. All measurements are performed at a controlled temperature $\mathrm{T}=20.0 \pm 0.5^{\circ}$ C.

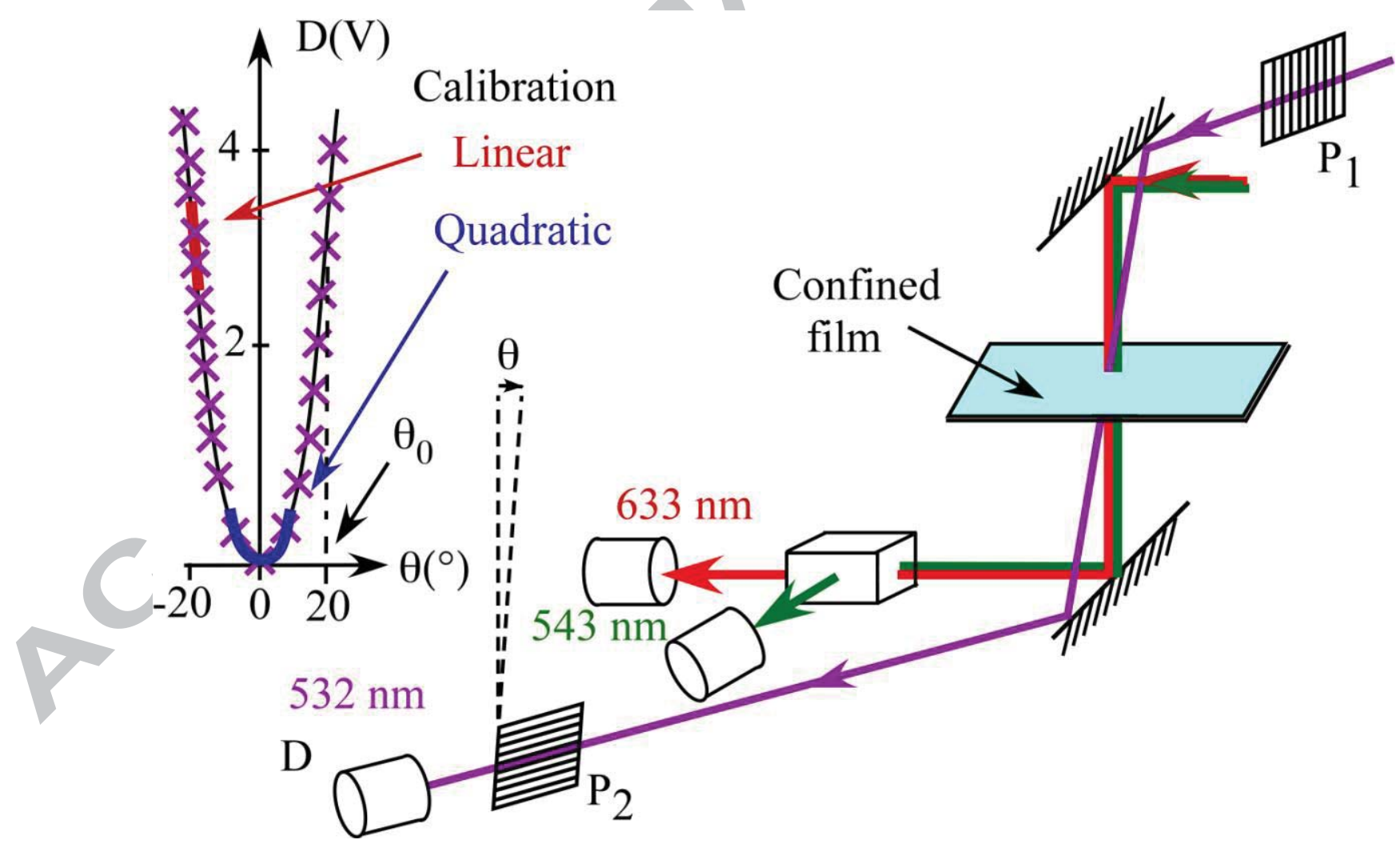

Fig. 1. Experimental set-up: The film liquid is confined between two glass slides. The optical activity is measured with a $532 \mathrm{~nm}$-laser between nearly crossed polarizers $\left(\mathrm{P}_{1}\right.$ and $\mathrm{P}_{2}$ ) via a photodiode (D) that has been calibrated just by rotating $\mathrm{P}_{2}, \mathrm{P}_{1}$ being fixed (insert). The purple crosses are experimental data; the error bars are in the crosses. Close to zero, the variation is quadratic (blue curve), whereas around $\theta_{0}$, the variation is linear with the rotation 
angle. The thickness of the film is measured with a red $(\lambda=633 \mathrm{~nm})$ and a green $(\lambda=543$ $\mathrm{nm})$ lasers.

Because of this bias $\theta_{0}$ between the polarizers, we benefit from the sensitivity of the signal on (D) that has a "linear" variation as $\theta$ varies, instead of a "quadratic" behavior when $\mathrm{P}_{1}$ and $\mathrm{P}_{2}$ are crossed (for $\theta_{0}=0$ ). A $1 \mathrm{~V}$ signal on the photodiode was found to correspond to a rotation of the polarizer $\theta=4.75^{\circ}$ around $\theta_{0}$. This also allows us to determine the sign of the optical activity. Indeed, optical activities of sugar soap films can be determined according to Biot's law [24], $\alpha=[\alpha] l c$, where $l$ is the length of the traversed optically active medium (in $\mathrm{dm}$ ), $c$ is the concentration in $\mathrm{g} / \mathrm{mL}, \alpha$ is the observed rotation angle (in ${ }^{\circ}$ ) and $[\alpha]$ is the specific optical activity (in ${ }^{\circ} \mathrm{cm}^{3} /($ g.dm)).

In order to eliminate possible artefacts due to linear dichroism we have rotated the two polarizers with the same angle. The measured optical rotation has remained unchanged, thus eliminating any linear dichroism. As for the issues raised by the light scattering on the optical rotation signal, the distance between the confined film and the photodiode D is one meter. This eliminates the contribution of the light scattered to the measured optical signal. The size of the laser spot (beam waist) is of the order of $1 \mathrm{~mm}$, compared with the size of the film that is of the order of $2 \mathrm{~cm}$. The laser spot only probes a small part of the film. However, we have changed its position and we did not notice any difference in the measured optical signals. Besides, we have performed micro Raman spectroscopy showing that the film is rather homogenous in terms of thickness or composition.

The $633 \mathrm{~nm}$ and $543 \mathrm{~nm}$ - lasers are used to measure the film thickness via the interference signal. We register the interference fringes of the two probe lasers versus time together with the rotation of the polarization due to the optical activity, both for a glucose + SDS film (see figure 2) and for a glucose film (see figure 3). The thinning is much faster in the beginning, when the liquid drop is flattened by the slide. The confinement induced by the two slides significantly slows down evaporation and fringes are still observed after several hours. We have to wait for at least three days to get a nearly total evaporation. However, we were not able to follow the thinning over such a long time. 


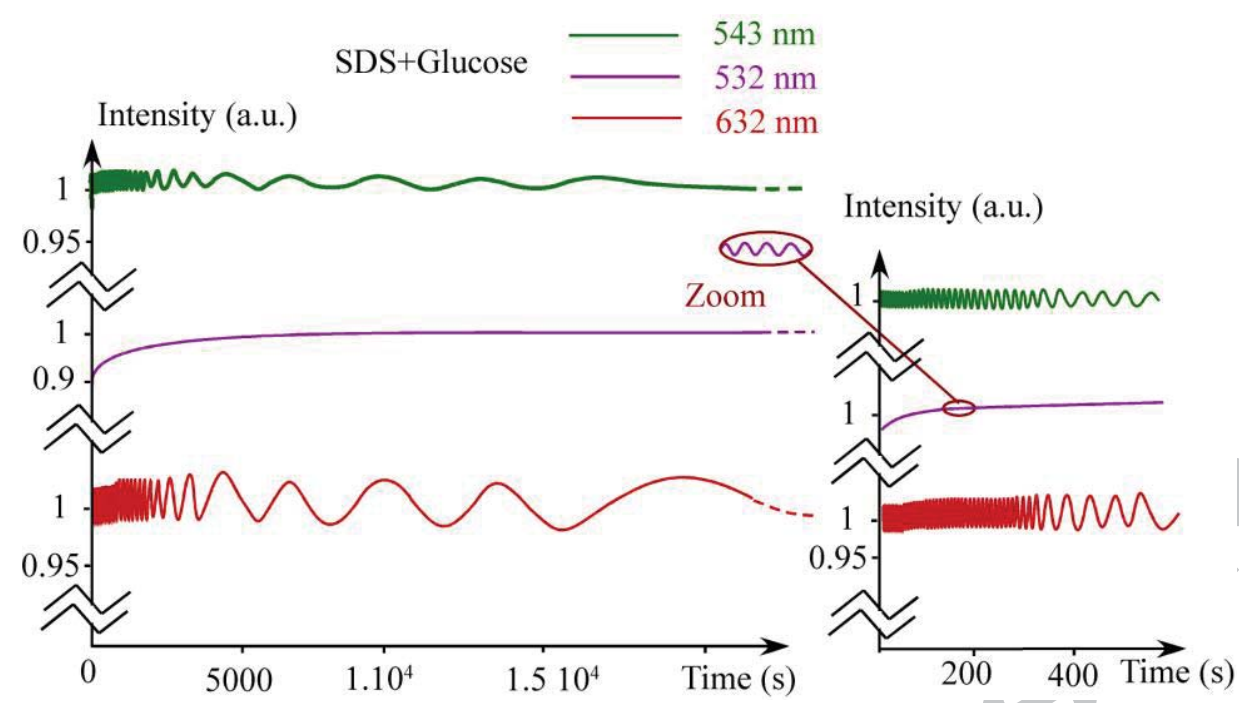

Fig. 2. Interference signal for a glucose + SDS film from the $632 \mathrm{~nm}$ laser (red curve) and $543 \mathrm{~nm}$ laser (green curve). Also shown in purple is the signal on (D). The signals have been normalized for all photodiodes and superposed. One can follow the film thinning over several hours.

Let us mention that the signal of the rotation of the polarization due to the optical activity at $\lambda=532 \mathrm{~nm}$ on $\mathrm{D}$ is also slightly modulated with time (see zoom). It corresponds to interference fringes due to the thinning of the film. However, this interference signal is much smaller than the signal of the optical activity and can be easily subtracted. Nevertheless, it could also help us to extract the thickness of the film, especially at the beginning of the thinning when the film is rather thick. We thus get a coarse estimation of the thickness considering the $543 \mathrm{~nm}$ and $532 \mathrm{~nm}$ lasers since their wavelengths are close, and a fine measurement considering the $543 \mathrm{~nm}$ and $632 \mathrm{~nm}$ lasers. 


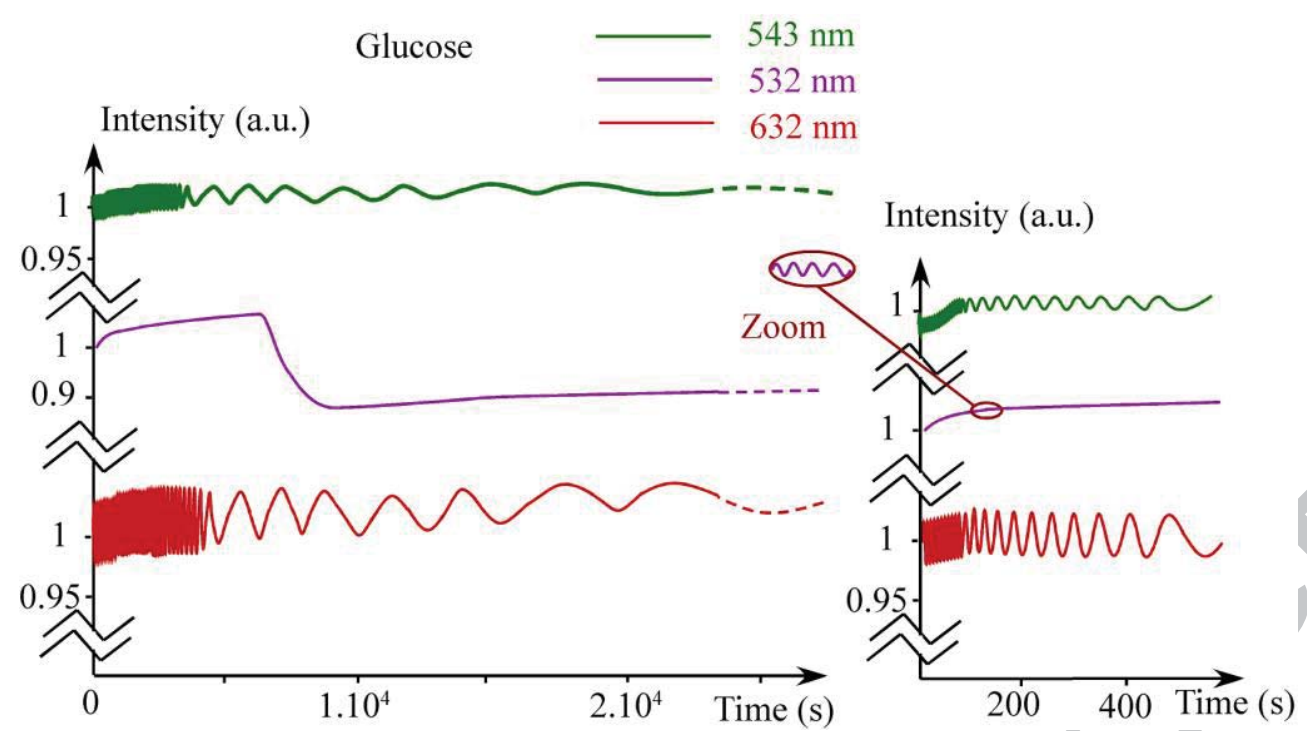

Fig. 3. Interference signal for glucose alone, from the $632 \mathrm{~nm}$ laser (red line) and $543 \mathrm{~nm}$ laser (green line) lasers. Optical activity signal is in purple. The signals have been normalized for all photodiodes and superposed.

\section{B. Computational details}

In order to get a clearer insight of the involved mechanisms, we combined experiments with molecular simulations. We performed molecular dynamic (MD) simulations of air/liquid and glass/liquid interfaces including SDS, $\mathrm{Na}^{+}$, glucose and water molecules. Water is modeled by means of the rigid non-polarizable TIP4P/2005 model [27], SDS and $\mathrm{Na}^{+}$are described by using the AMBER force field [28]. Partial charges of SDS are taken from Ref. [20]. Planar surface of silica is obtained by carving a surface in amorphous silica by means of the approach of Brodka and Zerda [29] leading to a surface with a density of surface silanol $(\mathrm{SiOH})$ groups of $7.5 \mathrm{SiOH} / \mathrm{nm}^{2}$. A snapshot is provided in figure 4. This matrix corresponds to a highly hydrated protonated silica pore $[29,30]$. Details on the framework building method and force field have been taken from elsewhere [31-33]. The axial length of silica membrane is $L_{z}=58 \AA$ and has been chosen to avoid the interactions between both interfaces i.e. to overcome size effects. MD simulations were conducted with two glass/liquid interfaces to use periodic boundary conditions along the normal of the interface i.e. into the $\mathrm{z}$ direction. Experimentally speaking the film is confined between two glass surfaces such that the film thickness is of the order of $10 \mu \mathrm{m}$. Simulations allow us to capture the liquid structure close to the silica surface while the confinement effect was not taken into account in our simulations. Although the silica matrix is subsequently kept rigid, 
rotation around the Si-O bond of the hydroxyl groups is allowed from the application of a bending potential between Si-O-H. Motions of hydrogen atoms of $\mathrm{SiOH}$ are performed from a stretching potential. Intramolecular parameters are given in [33]. Regarding to the airliquid phase, a bulk liquid phase is pre-equilibrated in the NpT statistical ensemble ( $\mathrm{N}$ is the number of molecules, $\mathrm{p}$ the pressure and $\mathrm{T}$ the temperature). Thereafter two empty boxes are added to create an air phase. Details of methodologies to model an air-liquid phase can be found elsewhere [20]. All MD (Molecular Dynamics) simulations have been carried out with the DL_POLY package [34] using a combination of the velocity-Verlet algorithm and the Nose-Hoover thermostat with a relaxation time of 0.5 ps [35]. MD simulations are performed using a time step of 0.002 ps to sample 50 ns (acquisition phase). To get a well compromise between computational times and statistical sampling 4900 water molecules, 20 SDS anionic surfactants, $20 \mathrm{Na}^{+}$cations and 20 glucose molecules are considered. In both cases glucose and glucose + SDS final dimensions are $\mathrm{L}_{z}=173 \AA$ and $\mathrm{L}_{\mathrm{z}}=179 \AA$ respectively. Surfacic molar concentrations $\left(\rho_{\text {surf }}\right)$ of glucose are $0.06 \mu \mathrm{mol} / \mathrm{m}^{2}$ and 0.02 $\mu \mathrm{mol} / \mathrm{m}^{2}$ for glucose alone and glucose + SDS, respectively. $\rho_{\text {surf }}$ of SDS is $0.02 \mu \mathrm{mol} / \mathrm{m}^{2}$ below the cmc concentration that corresponds to a total filling of the interface (these concentrations are calculated from the density profiles). Reproduction of experimental concentrations will lead to carry out large-scale MD simulations that will need an over expensive computational time. Let us mention that this work focuses on the interfacial phenomena located between 30 and $60 \AA$. Beyond $60 \AA$ the bulk phase is recovered. 


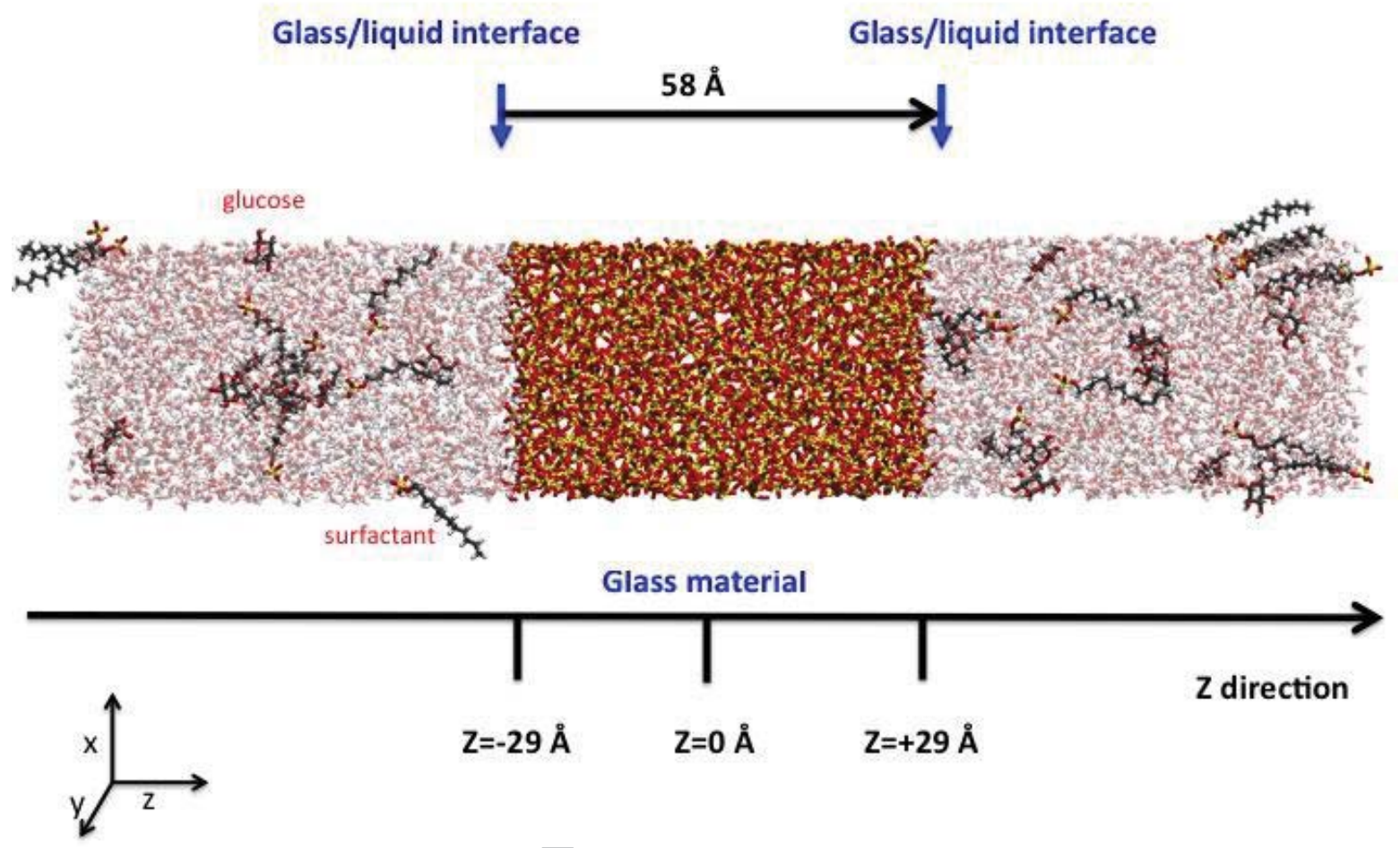

Fig. 4: Illustration of an initial configuration of water and glucose at both silica interfaces. Carbon, oxygen, hydrogen and silicon atoms are in black, red white, yellow colors respectively. For clarity SDS molecules were removed and water is transparent.

\section{Results and discussion}

\section{A. Optical rotation}

\section{A1. Glucose + SDS}

Figures 2 and 3 show interference fringes. Two consecutive fringes correspond to a thinning of the film of $\lambda / 2$. Comparing the fringes for the three wavelengths leads to a precise estimation of the film thickness for glucose and glucose + SDS. From the raw data on the photodiode D and according to its calibration, the optical rotation as a function of time can be deduced. As depicted in figure 5 the optical rotation of glucose + SDS solution varies linearly with the thickness. According to Biot's law, the glucose concentration in 
solution must be constant during film thinning. Thus during evaporation, the sugar molecules accumulate towards the film periphery in a way similar to the ring coffee effect [25]. This hypothesis is confirmed by Raman spectroscopy spectra during thinning where we found a much higher sugar concentration at the boundaries of the film.

Considering the slope of the angle of rotation, we find an optical activity $[\alpha]=$ $+2^{\circ} \cdot \mathrm{cm}^{3} /(\mathrm{g} . \mathrm{dm})$ that is in agreement with the optical activity of a bulk solution of sugar (with or without surfactant molecules). However, the extrapolation of the so-observed optical rotation of glucose + SDS towards zero crosses the $y$-axis at $+0.5^{\circ}$. Whereas the slope is linear with the thickness that corresponds to a bulk effect, this offset must correspond to a surface effect only. This means that there is a specific optical activity due to the glass/liquid interface alone. The glucose molecules in the presence of SDS molecules are probably organized differently at the interface, similar to what we found at the air/liquid interface [20]. However, for an air/liquid interface, there is clearly a change of the slope of the optical rotation versus thickness. The extrapolation of the experimental curves of reference [20] for thicknesses over $0.5 \mu \mathrm{m}$ towards zero leads to an offset of $+0.4^{\circ}$ for a high glucose concentration (400 g/L). This is quite different from the value of $+0.5^{\circ}$ we found here. If the optical activity is sensitive to coupling of glucose with SDS, it also depends on the nature of the interface. Additionally, the comparison between these two types of interface is restrictive because it has not been possible to obtain a thinner film than $4 \mu \mathrm{m}$ here. The evaporation process is nearly blocked due to the high confinement, contrarily to what we find for an air/liquid interface. 


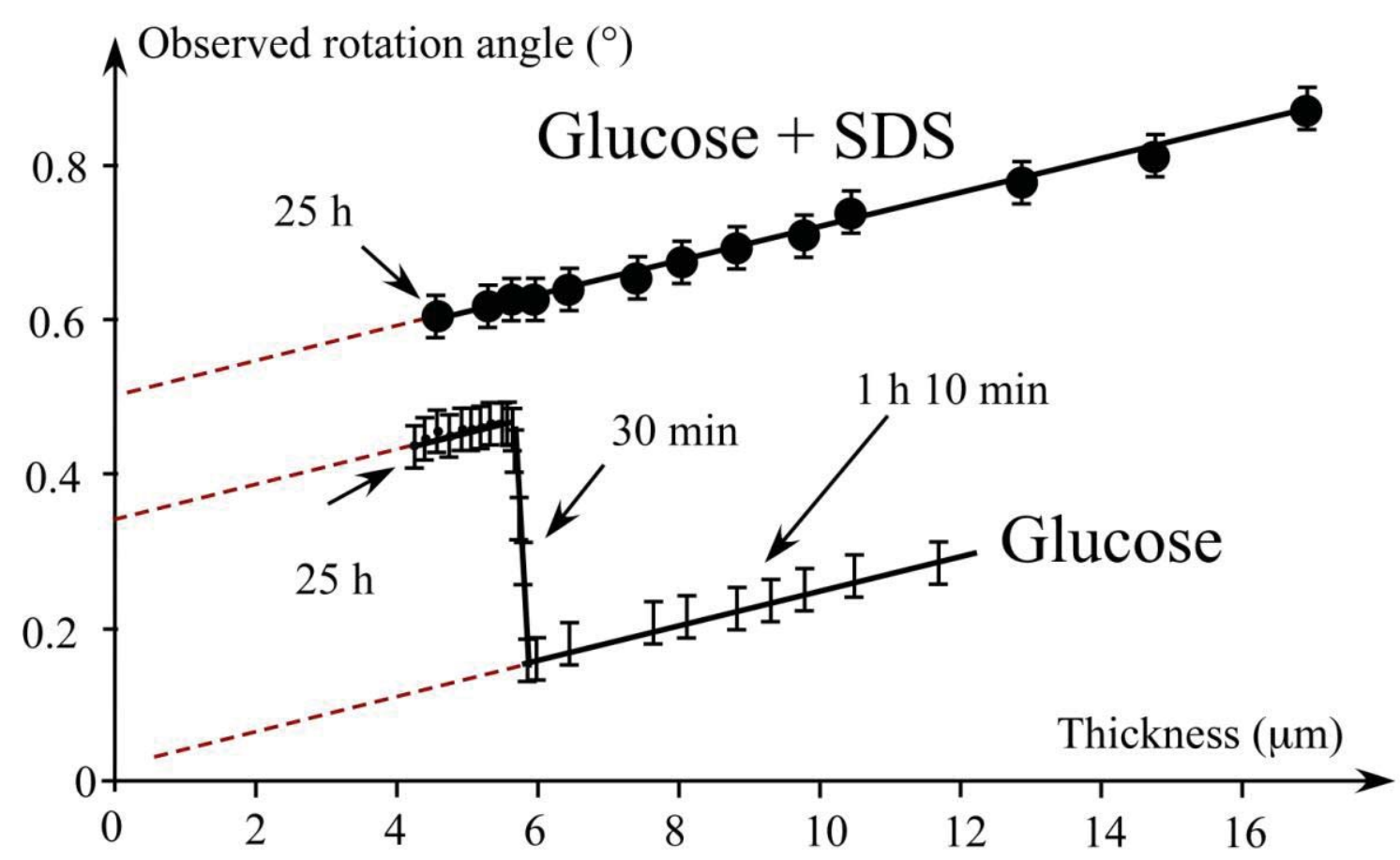

Fig. 5. Rotation angle for glucose (vertical bars) and glucose + SDS (plain circles) as a function of film thickness. We have registered the optical rotation, and the signal on the photodiode every $100 \mathrm{~ms}$. We could have plotted experimental points every $100 \mathrm{~ms}$. For the sake of clarity we have only selected some of them. The solid lines are the result of refinements of experimental data. The dotted lines correspond to extrapolation of these fits.

\section{A2. Glucose alone}

For glucose alone figure 5 shows a different optical behavior. At the beginning of the thinning, the so-observed rotation angle evolves linearly with the same slope as for the glucose + SDS and seems to be governed by the optical activity of the bulk solution alone. Then, for a thickness around $6 \mu \mathrm{m}$, the rotation of the polarization due to optical activity changes suddenly. This modification takes place after about one and a half hour of evaporation in a 30 minutes time interval and can due to a structural phase transition. In order to validate this rather sudden change of the optical rotation, we have performed the experiments more than ten times. Every time, the evolution is the same.

Then the rotation decreases with the same slope as at the beginning. This dynamics is governed by the optical properties of the bulk solution. However, the extrapolation of the experimental data towards zero leads to an offset value of $+0.35^{\circ}$. Further experiments with 
other glucose concentrations lead to the same offset, since the surface effect should saturate to the same value. There must have been a rearrangement of the glucose molecules at the glass/liquid interface. Yet, the offset has not the same value for glucose and glucose + SDS, meaning that the interfacial anchoring of the glucose molecules is different. Besides, we could not explain why this rearrangement for glucose molecules happened 90 min after the beginning of the experiment. It might be that glucose molecules at the glass/liquid interface diffuse more slowly in the absence of SDS to adopt their final conformation. To get a relevant standpoint of molecular organization close to the interface molecular dynamics simulations have been performed.

\section{B. Glass/liquid interface}

Figure 6 shows profile density along the z-direction (normal of the interface) of glucose alone and of glucose + SDS at the vicinity of the glass/liquid interface. Calculations of density profiles were performed by considering the centers of mass of molecules. In both cases an enhancement of density is found. Indeed, there is an arrangement of molecules close to the interface. As shown in figure 6, three layers for glucose are evidenced (figure 6a) and alternative layers for glucose and SDS (figure 6b). In figure 6, we can also notice that in the presence of SDS, the density of glucose decreases from $2.10^{-3}$ to $6.10^{-4}$ molecules/ $\AA^{3}$. For a same concentration in glucose, a lessening in interfacial glucose density seems to appear in the presence of SDS. This decrease suggests that the surfactants replace interfacial glucose molecules. This change in local density is probably independent of the initial concentration in glucose because SDS molecules absorb them at the silica surface to replace the interfacial glucose that is released in the bulk region. However, the total number of glucose molecules is constant between -30 $\AA$ and -60 $\AA$. The interfacial concentration of glucose molecules is thus the same with or without SDS and does not play a role on the optical activity.

Experimentally, we cannot probe thickness below $4 \mu \mathrm{m}$. When we invoke an interface effect on the optical activity, we mean an effect due to the rearrangement of the molecules in the vicinity of the interface, i.e. in the $100 \AA$ region, close to the interface, as shown in figure 6 . Besides, we have changed the glucose concentration of the solution but the offset remained the same. The value of the offset due to the molecules rearrangement near the interface does not thus depend on the glucose concentration. This clear anchoring of the glucose molecules at the glass/liquid interface can explain the offset of the observed 
rotation angle versus film thickness on figure 4. However, the simulations cannot explain the "phase transition" we observed. Indeed MD simulations sample motions in the time scale of picoseconds to nanoseconds.
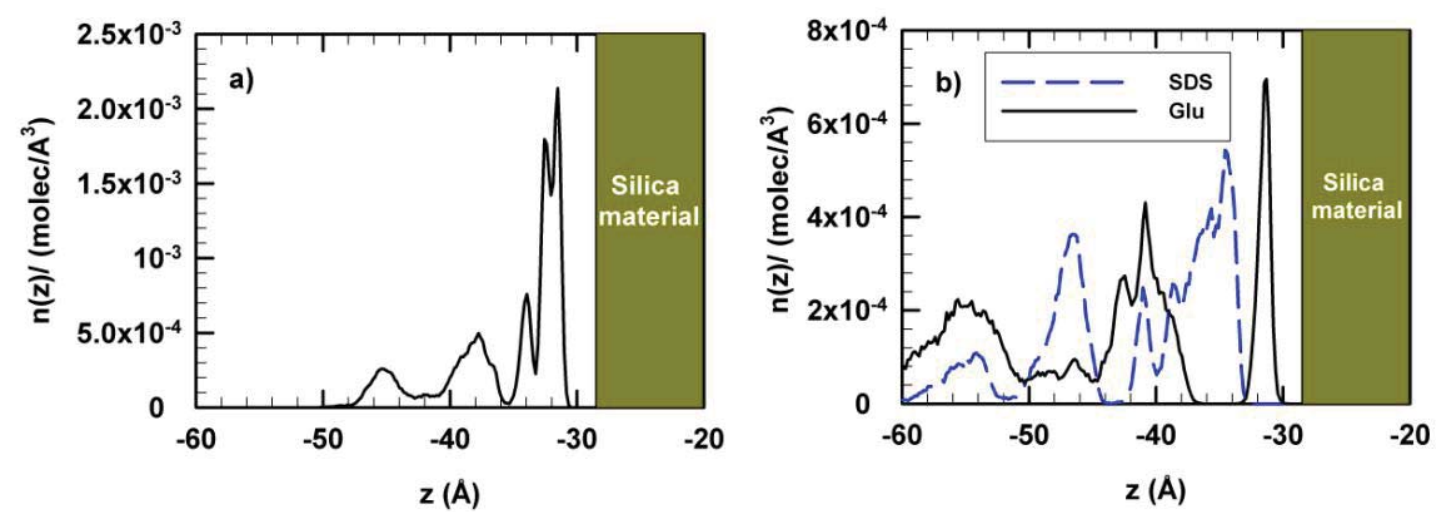

Fig. 6. Density profiles of glucose for glucose alone a) and glucose and SDS for glucose + SDS system b) in the z-direction that is normal to interfaces, figure 4 allowing location of center of system ( $\mathrm{z}=0 \AA$ ). Given the symmetry between both interfaces (see figure 4) the profiles have been reported only one interface. (Beyond -60 $\AA$ we found the bulk phase). The error bars are too small to be given. As example error bar on density profile of glucose is $1.1 \times 10^{-5}$.

Nevertheless, these simulations could give an insight of the reason why the offsets of the optical activity plots are different for glucose and glucose + SDS. On figure 7, we report the orientation of the dipole vector of the interfacial sugar molecules regarded to the normal of the interface i.e. along $\mathrm{z}$ axis. Whereas in the case of glucose alone, the dipoles are oriented perpendicular to the interface i.e. along the $\mathrm{z}$ axis $\left(\phi=0\right.$ or $\left.180^{\circ}\right)$, the dipoles of the glucose molecules are oriented at $\left(\phi=35^{\circ}\right)$ for glucose and SDS. This preferential orientation close to the interface could induce a new optical activity and could be then at the origin of the so-observed offset. 


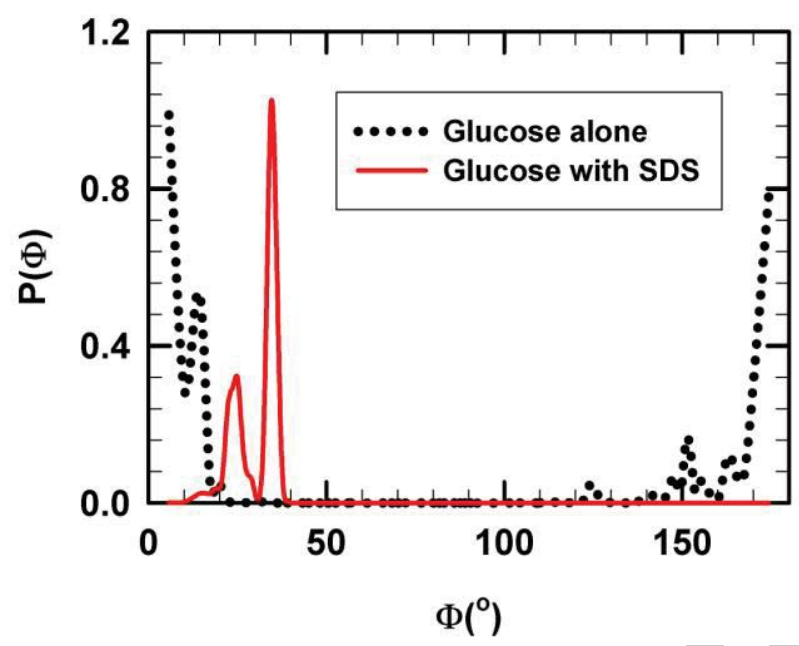

Fig. 7. Angular distribution of the dipole moment of glucose regarded to the normal of the glass/liquid interface. For $\phi=0$ or $180^{\circ}$, the dipoles are oriented perpendicular to the interface.

\section{Conclusions}

We have evidenced an optical activity in a confined thin film that does not tend towards zero when the thickness goes towards zero. This has been interpreted by a molecular arrangement of the sugar molecules at the glass/liquid interface due to their interfacial anchoring. This offset is different with or without the presence of surfactant molecules (SDS). It is usually admitted that dissolving sugar in a soapy solution provides stable and large films. However, contrarily to popular belief, it seems here that the lifetime should be mainly related to the interfacial structure and not to the rheological properties.

Molecular simulations have been performed to get an atomistic standpoint of the glass/liquid interface. By calculating the density profile of the center of mass of molecules for both glucose alone and glucose + SDS cases an interfacial anchoring of the glucose molecules have been highlighted. For glucose alone a layering structure was evidenced due to the excluded volume. A different structure was found for glucose + SDS system where alternated layers of glucose and SDS were evidenced looking like a local segregation. Furthermore, a preferential interfacial orientation of glucose molecules was highlighted in both cases. While a parallel orientation to the normal of the surface was found for the glucose alone the dipoles of the glucose molecules are slitghly tildted for glucose and SDS. 
This preferential orientation close to the interface could induce a new optical activity and could be then at the origin of the so-observed offset.

This molecular anchoring of the sugar molecules at the glass/liquid interface together with the work at the air/liquid interface, could shine some new light on the problem of glucose adsorption [36] and glucose interactions and sensing [37,38] in thin films at air/liquid or solid/liquid interfaces. In general, the optical activity can be an accurate parameter to understand the interfacial organization of the sugar in the growth of biofilms [39]. The simulations also showed a minor effect of water on the interfacial properties. Finally, the control of the drying process in nanometer-thick films would be a new useful tool towards a better manipulation of the polarization of light in optofluidic systems [40,41].

\section{Acknowledgements}

We acknowledge technical support from P. Chasles, and J.-R. Thébault, from A. Moréac for Raman spectra studies, and fruitful discussions with R. Lefort and D. Morineau. 


\section{References}

[1] H. J. Tong, J. P. Reid, D. L. Bones, B. P. Luo, and U. K. Krieger, Atmospheric Chemistry and Physics 11 (2011) 4739-4754.

[2] Comprehensive Chiroptical Spectroscopy, Vols.1\&2, N. Berova, P. L. Polavarapu, K. Nakanishi, R. W. Woody eds, Wiley, Hoboken, New Jersey, 2012.

[3] D. Sofikitis, L. Bougas, G. E. Katsoprinakis, A. K. Spiliotis, B. Loppinet, and T. Rakitzis, Nature 514 (2014) 76-79.

[4] B. M. Maoz, Y. Chaikin, A. B. Tesler, O. Bar Elli, O., Z. Fan, A. O. Govorov, and G. Markovich, Nano lett. 13 (2013) 1203-1209.

[5] I. Rea, P. Giardina, S. Longobardi, F. Porro, V. Casuscelli, I. Rendina, and L. De Stefano, J. R. Soc. Interface 9 (2012) 2450-2456.

[6] N. S. Oliver, C. Toumazou, A. E. G. Cass, and G. Johnston, Diabetic Med. 26 (2009) 197-210.

[7] L. Št’ovíčková, M. Tatarkovič, H. Logerová, J. Vavřinec, and V. Setnička, Analyst 140 (2015) 2266-2272.

[8] J. Chapiro, S. Sur, L. J. Savic, S. Ganapathy-Kanniappan, J. Reyes, R. Duran, S. C. Thiruganasambandam, C. R. Moats, M.-D. Lin, W. Luo, P. T. Tran, J. M. Herman, G. L. Semenza, A. J. Ewald, B. Vogelstein, and J. F. Geschwind, Clin. Cancer Res. 20 (2014) 6406-6417.

[9] K. D. Foster, J. E. Bronlund, and A. T. Paterson, J. Food Eng. 77 (2006) 997-1006.

[10] Y. Liu, B. Bhandari, and W. Zhou, J. Food Eng. 81 (2007) 599-610. 
[11] C. Ohkuma, K. Kawai, C. Viriyarattanasak, T. Mahawanich, S. Tantratian, R. Takai, and T. Suzuki, Food Hydrocolloids 22 (2008) 255-262.

[12] B. D. Cameron, H. W. Gorde, B. Satheesan, and G. L. Cote, Diabetes Technol. Ther. 1 (1999) 135-143.

[13] J. T. Suri, D.B. Cordes, F. E. Cappuccio, R. A. Wessling, and B. Singaram, Angew. Chem. Int. Ed. 42 (2003) 5857-5859.

[14] F. Chee, T. Fernando, Closed loop control of blood glucose, Springer-Verlag, BerlinHeidelberg, 2007.

[15] Y. H. Roos, Annu. Rev. Food Sci. Technol 1 (2010) 469-496.

[16] M. S. Steiner, A. Duerkop, and O. S. Wolfbeis, Chem. Soc. Rev. 40 (2011) 48054839.

[17] G. Pescitelli, Chirality 24 (2012) 718-724.

[18] P. L. Polavarapu and R. Vijay, J. Phys. Chem. A 116 (2012) 5112-5118.

[19] Y. Liu, R. Wang, and X. Zhang, Opt. express 22 (2014) 4357-4370.

[20] J. Emile, O. Emile, A. Ghoufi, A. Moréac, F. Casanova, M. Ding, and P. Houizot, J. Colloid Interface Sci. 408 (2013) 113-116.

[21] J. Emile, F. Casanova, G. Loas, and O. Emile, Soft Matter 8 (2012) 7223-7227.

[22] J. Emile, O. Emile, and F. Casanova, EPL 101 (2013) 34005.

[23] J. Emile, M. H. V. Werts, F. Artzner, F. Casanova, O. Emile, J.R.G. Navarro, and F. Meneau, J. Colloid Interface Sci. 383 (2012) 124-129.

[24] J. B. Biot, Mém. Acad. Sci. (France) 13 (1835) 39-175. 
[25] R. D. Deegan, O. Bakajin, T. F. Dupont, G. Huber, S. R. Nagel, and T. A. Witten, Nature 389 (1997) 827-829.

[26] A. Crivoi and F. Duan, Langmuir 29 (2013) 12067-12074.

[27] J. Abascal and C. Vega, J. Chem. Phys. 123 (2005) 234505.

[28] W. D. Cornell, P. Cieplak, C. I. Bayly, I. R. Could, K. M. Merz, D. Jr. Ferguson, D. Spellmeyer, T. Fox, J. W. Caldwell, and P. A. Kollman, J. Am. Chem. Soc. 117 (1995) 5179-5197.

[29] A. Brodka and T. Zerda, J. Chem. Phys. 104 (1996) 6319-6326.

[30] J. Puibasset and R. J.-M. Pellenq, J. Chem. Phys. 122 (2005) 094704.

[31] A. Ghoufi, I. Hureau, R. Lefort, and D. Morineau, J. Phys. Chem. C 115 (2011) 17761-17767.

[32] R. Renou, A. Ghoufi, A. Szymczyk, H. Zhu, J.-C. Neyt, and P. Malfreyt, J. Phys. Chem. C 117 (2013) 11017-11027.

[33] A. Ghoufi, D. Morineau, and R. Lefort, J. Chem. Theory Comput. 6 (2010) 32123222.

[34] I. Todorov, W. Smith, K. Trachenko, and M. Dove, J. Mater. Chem. 16 (2006) 19111918.

[35] M. P. Allen and D. Tildesley, Computer simulations of liquids, Oxford, 1987.

[36] S. Joshi, A. Rao, H. J. Lehmler, B. L. Knutson, and S. E. Rankin, J. Colloid Interface Sci. 428 (2014) 101-110.

[37] M. Khan and S. Y. Park, J. Colloid Interface Sci. 457 (2015) 281-288. 
[38] E. J. Grasso, R. G. Oliveira, and B. Maggio, J. Colloid Interface Sci. 464 (2016) 264276.

[39] P. Lembre, C. Lorentz, and D. P. Martino, The Complex World of Polysaccharides, in Tech, 2012, 371-392.

[40] H. Schmidt and A. R. Hawkins, Nat. photon. 5 (2011) 598-604.

[41] M. Oliva-Ramirez, A. Barranco, M. Löffler, F. Yubero, and A. R. González-Elipe, ACS nano. 10 (2016) 1256-1264. 


\section{Graphical abstract}
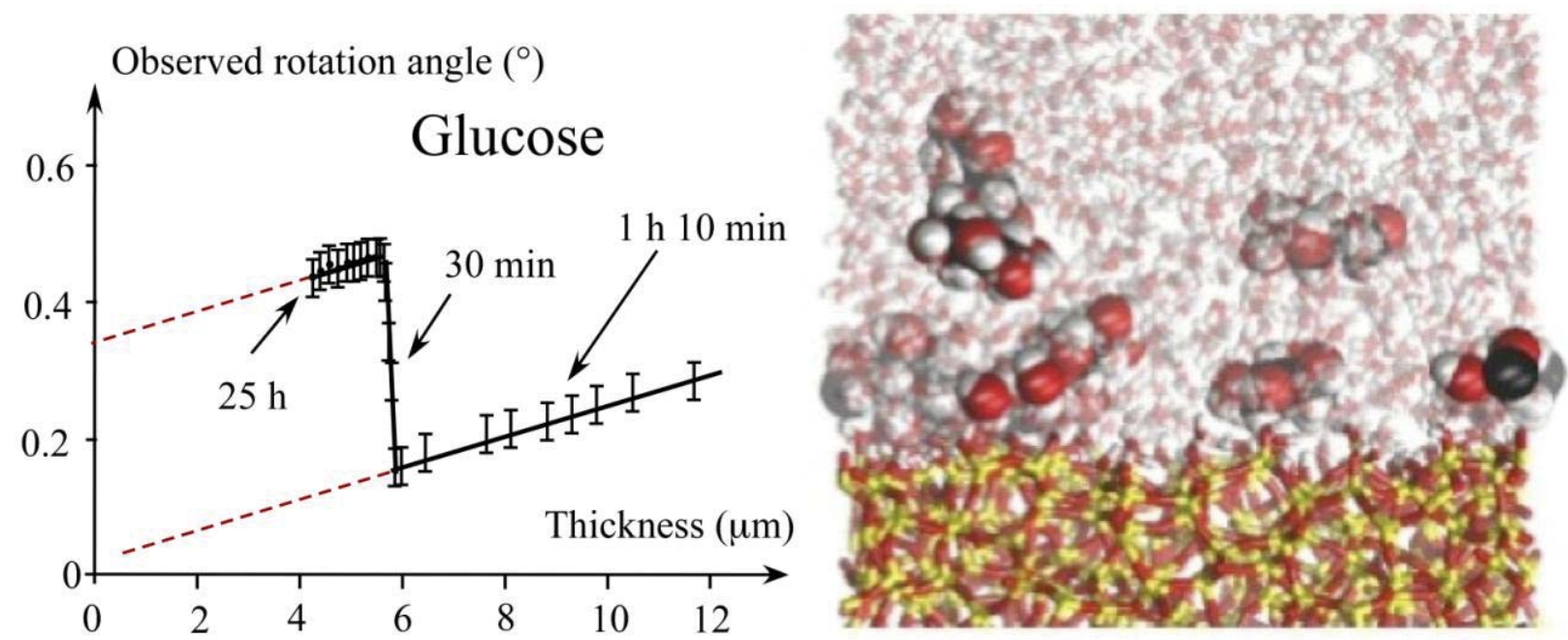

Optical activity and molecular anchoring of glucose molecules at the silica interface.

\section{Highlights}

- Optical activity of thin confined sugar films.

- Structure of sugar molecules at the glass/liquid interface. 